\title{
Intercurrence of Paratuberculosis in Intradermal Tuberculin Test Reactive Cattle
}

\author{
Mariana Assunção de Souza', Nicolle Pereira Soares², Alessandra Aparecida Medeiros-Ronchi², \\ Brendhal Almeida Silva ${ }^{3}$, Pedro Paulo Feitosa de Albuquerque ${ }^{4}$, \\ Rinaldo Aparecido Mota ${ }^{4}$ \& Anna Monteiro Correia Lima ${ }^{1}$
}

\begin{abstract}
Background: Bovine tuberculosis control programs are based on a standard diagnostic method, the intradermal test with purified protein derivatives, which is used to identify and eliminate diseased animals. Currently none of the tests available allow complete differentiation between infected and uninfected animals. The main limitations of the tests available are related to diagnostic sensitivity and specificity, which results in false positive reactions due to the existence of cross infections, and also false negative, inherent to the state of energy of some animals. The aim of this work was to study the intercurrence of paratuberculosis in tuberculosis reactive cattle by the comparative cervical test.

Materials, Methods \& Results: Three hundred and thirty four cattle were evaluated using the comparative cervical test (CCT) and serology for tuberculosis (TB) and paratuberculosis (PTB) ELISA IDEXX®. All of the animals testing positive by CCT were euthanized and necropsied. Fragments of lymph node, lung and intestine were collected and analyzed using histopathological techniques, with staining by Hematoxylin-Eosin (HE). Samples of lung and lymph nodes (retropharyngeal, submandibular, cervical and mediastinal) of the animals testing positive by CCT were evaluated using qPRC for M. bovis, and intestinal and mesenteric lymph nodes using PCR for PTB. Of the 334 cattle evaluated using the comparative cervical test, 16 were considered positive. No lesions suggestive of tuberculosis were found in the macroscopic inspection of the carcasses. The most evident anatomical and pathological finding was a thickening of intestinal mucosa, found in 12 of the 16 cattle submitted to necropsy. No microscopic lesions suggestive of TB were identified nor was the presence of $M$. bovis detected by qPCR. The main histopathological findings were observed in the small intestine and mesenteric lymph nodes and identified as enteritis, lymphangitis, lymphangiectasia and granulomatous lymphadenitis. In the intestine the changes are characterized by dilated and inflamed lymphatic vessels and intense inflammatory infiltrate on the mucosa and submucosa. Of the 334 serum samples evaluated, the M. bovis ELISA Antibody Test (IDEXX®) identified 17 positive animals. All the cattle considered positive by $M$. bovis ELISA were considered negative by CCT. In the samples from nine animals (9/16), DNA from M. avium subsp. paratuberculosis (MAP) was identified and in twelve carcasses (12/16) lesions characteristic of PTB were found, which were subsequently confirmed by histopathological techniques. In another nine animals of the herd anti-MAP antibodies were detected. None of those that tested positive by PTB ELISA were reactive by CCT.

Discussion: Animals considered positive by TB ELISA that were not positive in the intradermal test does not mischaracterize the clinical picture of the disease. Considering the inverse relationship between cell-mediated and humoral responses to M. bovis, the intradermal test and the serological tests are designed to measure different immunological responses, which develop during different stages of infection. The progress of the cellular immunological response to humoral immunity occurs in the most advanced stages of tuberculosis. Of the 16 cattle considered positive by CCT, 12 animals presented macroscopic and histological lesions suggestive of PTB and DNA from MAP was detected in nine. Although it is the official test for the control of TB in different countries, the intradermal test with PPD has presented limitations, primarily related to specificity. M. avium subsp. Paratuberculosis is considered the main cause of false positive reactions in the intradermal test. The PPD bacterial extract is a complex mixture of proteins, lipids, sugars and nucleic acids, and many of these components are also shared by numerous species of mycobacteria (tuberculous or not).
\end{abstract}

Keywords: cattle, Mycobacterium bovis, Mycobacterium avium subsp. paratuberculosis, serology, tuberculosis. 


\section{INTRODUCTION}

Bovine tuberculosis (TB) is a disease of economic and zoonotic importance, caused by bacterial agents of Mycobacterium tuberculosis complex, primarily M. bovis [11]. In addition, the genus Mycobacterium encompasses the Mycobacterium avium-intracellulare complex, formed by nontuberculous strains including M. avium subsp. paratuberculosis (MAP) which causes granulomatous enteritis, characterized by chronic diarrhea and loss of production [5,27].

The quality of the diagnostic tests used to detect animals infected with $M$. bovis has always been a challenge for the control of TB. The intradermal TB test is the most commonly used method in the world for detecting cattle with tuberculosis [22]. One of the main limitations of the in vivo and in vitro diagnosis of tuberculosis has been cross-reactivity, with responses induced by other nonspecific reactions with mycobacteria, most importantly MAP, considered the main cause of false positive reactions in the intradermal test [1].

To ensure control and reduce the prevalence of $\mathrm{TB}$, diagnostic tests that are more sensitive and specific are needed to improve control strategies and make strides toward eradication. In addition, more studies are needed that draw a correlation between pathological changes and the results of the diagnostic tests in animals coinfected with M. bovis and MAP under field conditions. Consequently, the aim of this work was to study the intercurrence of paratuberculosis in cattle reactive by the comparative cervical test for tuberculosis.

\section{MATERIALS AND METHODS}

The study was conducted in a dairy farm located in the municipality of Uberlândia, in the state of Minas Gerais. Holstein cattle of both sexes, aged between six months and 20 years, were evaluated.

A total of 334 cattle were examined using comparative cervical test (CCT), in line with recommendations by the National Program for the Control and Eradication of Brucellosis and Tuberculosis (PNCEBT) [6].

All the cattle that tested positive by comparative cervical test $(\mathrm{CCT})^{1}$ were euthanized and necropsied; carcasses and viscera were evaluated macroscopically, and the lesions recorded and photographed. During the inspection of the carcasses, lesions suggestive of paratuberculosis were observed, prompting a differential diagnosis of the disease. Fragments of approximately
$1.5 \mathrm{~cm}$ of lung, intestine, retropharyngeal, submandibular, cervical, mediastinal and mesenteric lymph nodes were collected and stored in sterilized plastic vials, containing $10 \%$ formaldehyde. The material was identified and sent to the Laboratory for Animal Pathology at UFU. The samples were dehydrated, diaphonized and paraffinized. Histological slides were submitted to Hematoxylin-Eosin staining ${ }^{2}$.

Samples of lung, retropharyngeal, submandibular, cervical and mediastinal lymph node of the animals testing positive by CCT were frozen and sent to the National Livestock Laboratory (LANAGRO) of the Ministry of Agriculture, Livestock and Food Supply (MAPA), in Pedro Leopoldo, MG. DNA extraction was performed using DNeasy Blood \& Tissue kit ${ }^{3}$ following manufacturer's instructions, without modification. Samples of the extracted DNA were submitted to quantitative PCR (qPCR) in the thermal cycler QuantStudio 7 Flex ${ }^{\text {TM }}$ Real-Time PCR System ${ }^{4}$ (Life Technologies, USA). The amplification reaction was performed according to Moura et al. [19].

For all the 334 cattle evaluated using CCT, $5 \mathrm{~mL}$ of blood was collected by coccygeal venipuncture. The material was transported under refrigeration $\left(2^{\circ} \mathrm{C}\right.$ to $\left.8^{\circ} \mathrm{C}\right)$ to the Laboratory of Infectious and Contagious Diseases (LADOC) of the School of Veterinary Medicine (FAMEV) at UFU. For the serological diagnosis of TB, the IDEXX $^{\circledR 5}$ Mycobacterium bovis ELISA Antibody Test was used. The test was performed in the LADOC - FAMEV - UFU, following instructions by the manufacturer.

For the serological diagnosis of paratuberculosis, the ELISA Mycobacterium avium subsp. paratuberculosis Antibody Test IDEXX ${ }^{\circledR 5}$ was used on all 334 samples. The test was performed at LANAGRO in Pedro Leopoldo, MG, following instructions by the manufacturer.

Samples from the intestine, ileocecal valve and mesenteric lymph nodes of the animals testing positive by CCT, were collected in sterilized vials and stored in an ultrafreezer at $-80^{\circ} \mathrm{C}$. The material was shipped under refrigeration to the Laboratory of Bacterioses of the Department of Preventive Veterinary Medicine at the Federal Rural University of Pernambuco. For the extraction of DNA was used the kit Wizard ${ }^{\circledR}$ SV Genomic DNA Purification System6. The extracted DNA was amplified to a final volume of $15 \mu \mathrm{L}$ containing: $5 \mu \mathrm{L}$ of genomic DNA; $0.5 \mu \mathrm{L}$ of the primers specific for IS 900 at 20 pMol (DF: 5'-GACGACTCGACCGCTAATTG-3' and DR-1: 5' - CCGTAACCGTCATTGTCCAG-3'); 
$2.75 \mu \mathrm{L}$ of Mili-Q ultrapure water and $6.25 \mu \mathrm{L}$ of MasterMix ${ }^{3}$ according to the protocol from the supplier. The thermal profile of the reactions was obtained using a New XP Thermal Cycler ${ }^{1}$ with an initial denaturation at $96^{\circ} \mathrm{C}$ for $5 \mathrm{~min}$, followed by 35 cycles at $95^{\circ} \mathrm{C}$ for 1 min each, annealing at $58^{\circ} \mathrm{C}$ for $1 \mathrm{~min}$ and extension at $72^{\circ} \mathrm{C}$ for $3 \mathrm{~min}$, and a final cycle of $72^{\circ} \mathrm{C}$ for $10 \mathrm{~min}$. The product amplified by $99 \mathrm{pb}$, corresponding to the DNA of MAP, was detected by agarose gel electrophoresis at $2 \%$, stained with blue-green 1 and viewed using ultraviolet light and photo documented [22].

\section{RESULTS}

Of the 334 cattle evaluated using the comparative cervical test, 16 were considered positive. The oscillation of the reactions in the reactive animals ranged from 0.5 to $4 \mathrm{~mm}(\Delta \mathrm{A}) ; 4.3$ to $8.8 \mathrm{~mm}(\Delta \mathrm{B})$ and 4 to $5.2 \mathrm{~mm}(\Delta \mathrm{B}$ $-\Delta \mathrm{A})$. In all, 16 cattle positive by CCT were euthanized.

No lesions suggestive of tuberculosis were found in the macroscopic inspection of the carcasses. The most evident anatomical and pathological finding was a thickening of intestinal mucosa, found in 12 of the 16 cattle submitted to necropsy. The surface was irregular, reddish and cerebroid in appearance, with pronounced transverse folds (Figure 1). The cecum also presented a thickening of the mucosa and had polypoid-like nodules. The mesenteric and ileocecal lymph nodes presented increased volume, with a darker appearance in the medullary region. Whitened nodules in the cortical region of the lymph nodes were found in 3 animals, which presented an accumulation of milky liquid in the medullary sinus (Figure 2). In 5 of the carcasses, the spleen was voluminous and pulposus. Prominent lymphoid follicles were observed upon cutting; and no macroscopic alterations were observed in the other organs.
The main histopathological findings were observed in the small intestine and mesenteric lymph nodes and identified as enteritis, lymphangitis, lymphangiectasia and granulomatous lymphadenitis. In the intestine the changes are characterized by dilated and inflamed lymphatic vessels and intense inflammatory infiltrate on the mucosa and submucosa slide itself (Figure 3), composed of lymphocytes, epithelioid macrophages, plasmocytes and eosinophils; hyperplasia of calciform glands; and muscular hypertrophy. In the mesenteric lymph nodes granulomatous nodular formations surrounded by inflammatory cells (lymphocytes and plasmocytes) were observed; and caseous necrosis and calcification (Figure 4). Note that Figure 4 shows the microscopic appearance of Figure 2.

Of the 334 serum samples evaluated, the $M$. bovis ELISA Antibody Test (IDEXX ${ }^{\circledR}$ ) identified 17 positive animals. All the cattle considered positive by M. bovis ELISA were considered negative by CCT.

Of the tissue samples (lung, retropharyngeal, submandibular, cervical and mediastinal lymph nodes) from the 16 cattle considered positive by CCT, M. bovis DNA was not detected in qPCR M. bovis.

Of the 334 serum samples evaluated, the $M$. avium subsp. paratuberculosis ELISA antibody test $\left(\right.$ IDEXX $^{\circledR}$ ) detected antibodies anti-MAP in 9 animals. All of the cattle considered positive by PTB ELISA were negative by CCT for tuberculosis. In these animals the reaction to avian $\operatorname{PPD}(\triangle \mathrm{A})$ ranged from 0.5 $\mathrm{mm}$ to $2.7 \mathrm{~mm}$.

Of the samples of intestine and mesenteric lymph nodes evaluated by PCR, collected from the 16 cattle reactive by CCT, 9 samples amplified the specific genetic sequence IS900 of $M$. avium subsp. paratuberculosis, for a total of 9 animals considered positive by PCR for PTB.

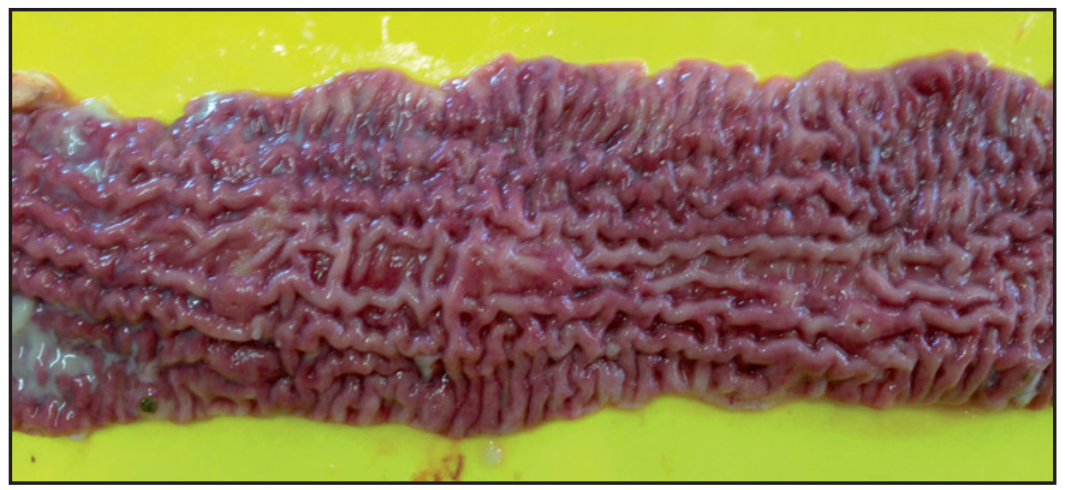

Figure 1. Thickened duodenal mucosa with reddish, wrinkled and/or cerebroid appearance, from a cow considered positive by the comparative cervical test for tuberculosis. 

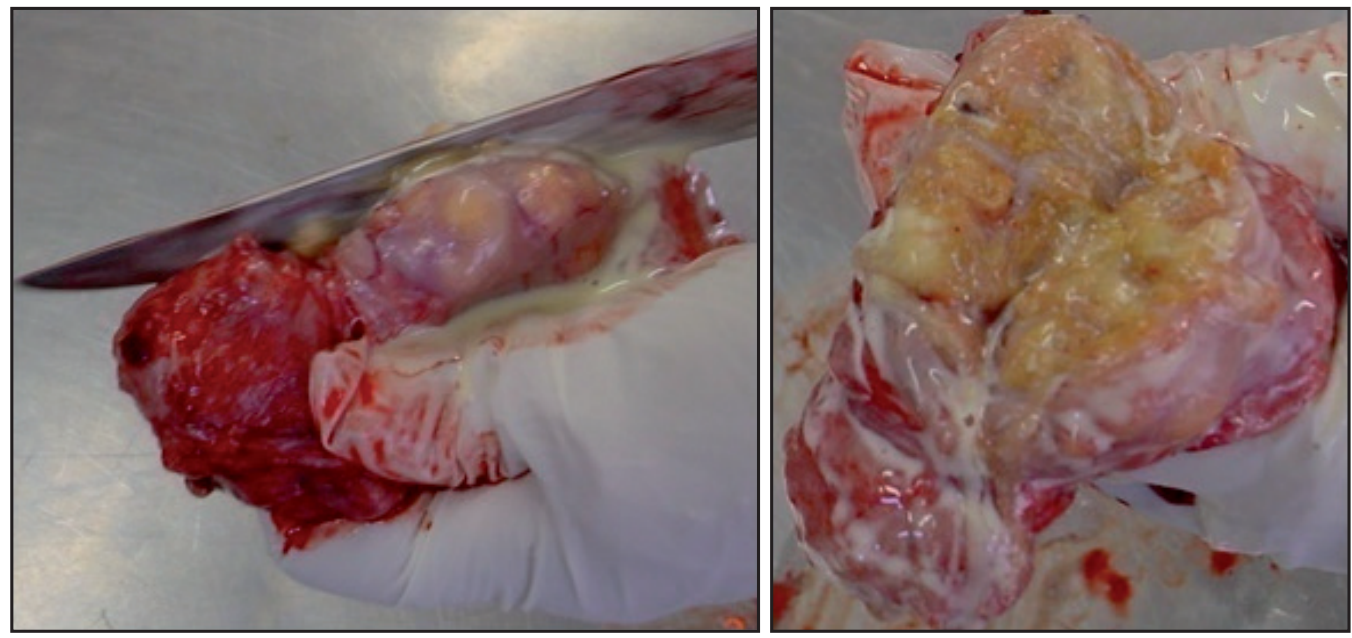

Figure 2. Mesenteric lymph node with irregular and whitened multi-nodular areas in the cortical region and the presence of liquid of milky appearance, from a cow considered positive by the comparative cervical test for tuberculosis.

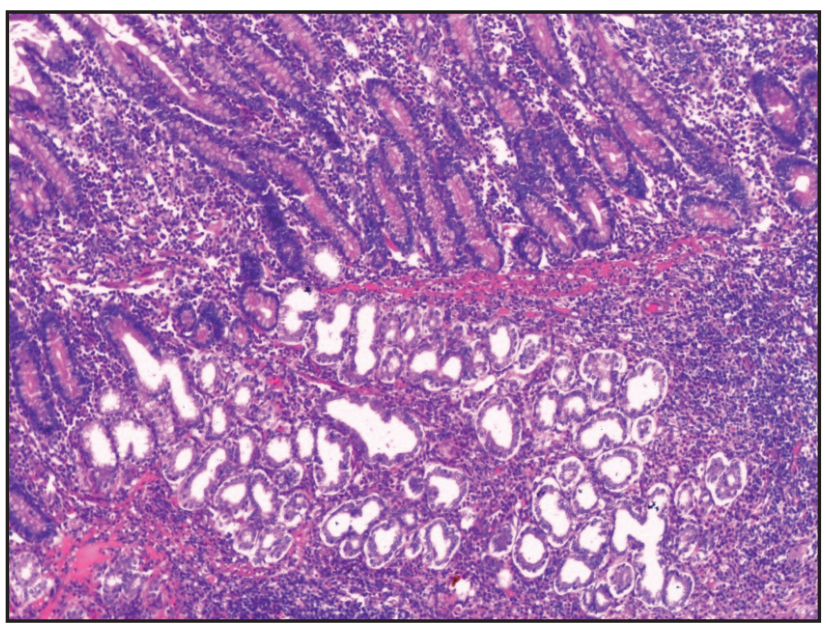

Figure 3. Photomicrograph of the small intestine with mucosa and submucosa presenting inflammatory infiltrate [HE; $4 \mathrm{x}]$.

\section{DISCUSSION}

Considering the positive results in the TB ELISA and CCT tests, the prevalence of cattle with tuberculosis in the herd was $9.8 \%$. The presence of anti- $M$. bovis antibodies in 17 cattle reinforces the occurrence of tuberculosis in the herd evaluated. The fact that the animals considered positive by TB ELISA were not considered positive in the intradermal test does not mischaracterize the clinical picture of the disease. Considering the inverse relationship between cell-mediated and humoral responses to $M$. bovis, the intradermal test and the serological tests are designed to measure different immunological responses, which develop during different stages of infection [8]. The progress of the cellular immunological response to humoral immunity occurs in

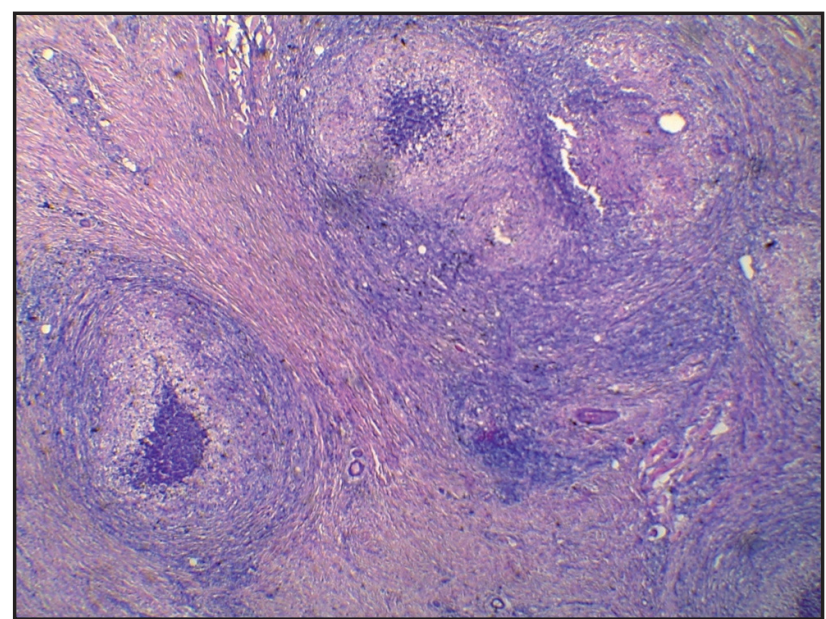

Figure 4. Photomicrograph of mesenteric lymph node with presence of granuloma [HE; 4x].

the most advanced stages of tuberculosis. For this reason, the assays based on antibodies are useful as complementary tests to the assays based on cellular response, aiding in the identification of cattle in more advanced stages of the disease, with false-negative results in the TB test, due to the stage of anergy of the animals $[25,26]$.

Of the 16 cattle considered positive by CCT, 12 animals presented macroscopic and histological lesions suggestive of PTB and DNA from MAP was detected in nine. Although it is the official test for the control of TB in different countries, the intradermal test with PPD has presented limitations, primarily related to specificity [16]. M. avium subsp. paratuberculosis is considered the main cause of false positive reactions in the intradermal test [5]. 
The PPD bacterial extract is a complex mixture of proteins, lipids, sugars and nucleic acids, and many of these components are also shared by numerous species of mycobacteria (tuberculous or not). This antigenic complexity is probably the main cause of the occurrence of nonspecific reactions in the TB tests [17]. MNT can coexist in the herd or in the same animal, leading to diagnostic errors at the individual and herd level [5].

The presence of macroscopic and histological lesions suggestive of PTB, and the amplification of genetic material from MAP confirm the occurrence of the disease in the herd studied. Of those testing positive, all were female, aged between 3 and 9 years and none of the animals had a clinical history of the disease. The absence of clinical signs does not discard the presence of infection in the herd, in these cases it is necessary to combine diagnostic techniques to confirm infected animals [18]. The clinical manifestations are generally observed after 3 years of age, although MAP can remain incubated for a period that ranges from 6 months up to 15 years, resulting in late clinical signs $[13,15]$.

The thickening of the intestinal mucosa, changes in lymphatic vessels of the mesentery and in the mesenteric lymph nodes, which histologically correspond to enteritis, lymphangitis and granulomatous lymphadenitis, are usually described in bovine paratuberculosis [9]. These lesions were observed in the animals necropsied in different degrees of intensity. The variation in the intensity of the lesions is related to the stage of the infection, immunity of the animal, the susceptibility of the host and the strain of MAP [24]. The detection of MAP DNA confirms the etiology of the paratuberculosis in the herd studied. Although there is no test today that is sufficiently capable of detecting all infected animals, the diagnosis of paratuberculosis requires a multidisciplinary focus on clinical and pathological manifestations, immunological response and detection of the etiological agent [14].

The paratuberculosis ELISA test detected anti-Map antibodies in nine animals $(2.7 \%)$, which were all negative by CCT and none of them presented clinical manifestations suggestive of paratuberculosis. These animals represent an important category in the control of paratuberculosis, since in addition to not presenting clinical signs, they intermittently eliminate the agent into the environ- ment and maintain the infection in the herd [9]. The sensitivity of the test depends on the stage of infection. When the disease is in the initial stages or latent, the sensitivity is low, approximately $15 \%$, and tends to increase (75\%) with the development of the disease to the clinical or terminal phase $[10,23]$. Although the serological tests did not present sufficient accuracy for an individual diagnosis, they are efficient in determining infected herds and allow an estimation of the extent of the problem in the suspected farm [20].

There is also the possibility of co-infection of TB and PTB in the herd studied. Mixed infections of $M$. avium (including MAP) and M. bovis have already been reported. Seva et al. [21], with the objective of characterizing the immune response and the profile of lesions in herds infected with M. bovis and MAP, identified that co-infected cattle, which present small granulomas or initial TB lesions, were considered positive by PCR and serology for PTB, although no lesion was identified for PTB. While the animals who had extensive TB lesions also presented diffuse lesions for PTB, demonstrating that there is a positive correlation with the seriousness of the pathology in animals co-infected with TB and PTB. Byrne et al. [7], found robust associations between TB herd breakdown episode risk and concurrent MAP infections at the herd level. This indicated that breakdown herds exhibited a higher risk of co-infection than sampled herds which were not experiencing a breakdown.

The intercurrence of cattle reactive by TB test with paratuberculosis was also reported [3]. The authors detected granulomatous lesions associated with the infection by MAP in $28 \%$ (26/91) of the cattle considered positive by the TB test. In the necropsy of the cattle considered positive by TB test it was also observed that the granulomas were located exclusively in the interfollicular areas of the lymphoid tissue in $16.4 \%$ (15/91) of the animals. Other granulomas were identified in the slide of the ileocecal valve itself in $12 \%$ (11/91) of the cattle. Using an immunohistochemical technique the authors detected MAP in 92\% (24/26) of the animals with PTB lesions. It is important to emphasize that no clinical signs of PTB were found in any of these animals, as with the present study. The detection of false positive results with the TB test is important particularly in areas where there is a relatively low 
incidence of TB and can be attributed, partly, to the cross immunological reactivity with other infections by mycobacteria, especially MAP [3].

Other studies indicate that co-infection by $M$. bovis and MAP lead not only to false positive results, but also to false negative results [4]. In experimental co-infections in which $M$. avium subsp. avium was administered previous to $M$. bovis, the animals did not react to the TB test the same way as when infected only with M. bovis [2]. In this case, these results may represent an obstacle to TB eradication programs, since false-negative animals may not be detected with the intradermal test, thus serving as an important source of infection from the disease. This is why, in practice, if the objective is to eradicate the disease, the ability of a diagnostic test to detect all infected animals (not missing truly infected animals with false-negative results) is more important than the error caused by the detection of false positive results [12,21]. Although they are not $100 \%$ specific, countries that have been able to eradicate TB used intradermal TB test as a basic tool in their sanitary programs. This is undoubtedly the best test for herds in which the disease is endemic and in controlled herds.

\section{CONCLUSION}

Under the field conditions studied, infection by M. avium subsp. paratuberculosis may have caused nonspecific reactions in the intradermal TB test. Despite the limitations regarding the specificity of the intradermal test, from an epidemiological perspective, it is the best test available for the detection of herds with tuberculosis.

\section{MANUFACTURERS}

${ }^{1}$ Instituto de Tecnologia do Paraná. Curitiba, PR, Brazil.

${ }^{2}$ Labsynth Produtos para Laboratórios Ltda. Diadema, SP, Brazil.

${ }^{3}$ Qiagen. Hilden, NW, Germany.

${ }^{4}$ Thermo Fisher Scientific Inc. Waltham, MA, USA.

${ }^{5}$ IDEXX Laboratories Inc. Westbrook, ME, USA.

${ }^{6}$ Promega Corporation. Madison, WI, USA.

Funding. The author(s) thank the financial support from CAPES (Coordination of Improvement of Higher-Level Personnel), CNPq - no. 64/2008 (National Council for Scientific and Technological Development).

Ethical approval. This research was approved by the Committee for Ethics in the Use of Animals (CEUA) at the Federal University of Uberlândia, under protocol n. 066/14.

Declaration of interest. The author(s) declared no potential conflicts of interest with respect to the research, authorship, and/or publication of this article.

\section{REFERENCES}

1 Aranaz A., DeJuan L., Bezos J., Alvarez J., Romero B., Lozano F., Paramio J.L., López-Sanchez J., Mateos A. \& Domínguez L. 2006. Assessment of Diagnostic Tools for Eradication of Bovine Tuberculosis in Cattle Co-infected with Mycobacterium bovis and M. avium subsp. paratuberculosis. Veterinary Research. 37(4): 593-606.

2 Amadori M., Tagliabue S., Lauzi S., Finazzi G., Lombardi G., Telo P., Pacciarini L. \& Bonizzi L. 2002. Diagnosis of Mycobacterium bovis Infection in Calves Sensitized by Mycobacteria of the avium/intracellulare Group. Journal of Veterinary Medicine. B, Infectious Diseases and Veterinary Public Health. 49(2): 89-96.

3 Balseiro A., Prieto J.M., Espí A., Perez V. \& Garcia-Marín J.F. 2003. Presence of Focal and Multifocal Paratuberculosis Lesions in Mesenteric Lymph Nodes and the Ileocaecal Valve of Cattle Positive to the Tuberculin Skin Test. Veterinary Journal. 166(2): 210-212.

4 Biet F., Boschiroli M.L., Thorel M.F. \& Guilloteau L.A. 2005. Zoonotic Aspects of Mycobacterium bovis and Mycobacterium avium intracellulare Complex (MAC). Veterinary research. 36(3): 411-436.

5 Biet F. \& Boschiroli M.L. 2014. Non-tuberculous Mycobacterial Infections of Veterinary Relevance. Research in Veterinary Science. 97: 69-77.

6 Brazil. Ministério da Agricultura Pecuária e Abastecimento. Programa Nacional de Controle e Erradicação da brucelose e tuberculose - PNCEBT. 2006. (National Program for the Control and Eradication of Brucellosis and Tuberculosis - PNCEBT). Brasília: MAPA/DAS/DAS, 184p.

7 Byrne A.W., Grahan J., Milne G., Guelbenzu-Gonzalo M. \& Strain S. 2019. Is there a Relationship Between Bovine Tuberculosis (bTB) Herd Brekdown Risk and Mycobacterium avium subsp. paratuberculosis Status? An Investigation in bTB Chronically and Non-chronically Infected Herds. Frontiers in Veterinary Science. 6: 30 1-11.

8 Chapinal N., Elkin B.T., Joly D.O., Shumaker B.A. \& Stephen C. 2012. Agreement Between the Caudal Fold Test and Serological Tests for the Detection of Mycobacterium bovis Infection in Bison. Preventive Veterinary Medicine. 105(4): 326-330. 
9 Chiodini R.J., VanKruiningen H.J. \& Merkal R.S. 1984. Ruminant Paratuberculosis (Johne's disease): The Current Status and Future Prospects. The Cornell Veterinarian. 74(3): 218-262.

10 Collins M.T., Gardner I.A., Garry F.B., Roussel A.J. \& Wells S.J. 2006. Consensus Recommendations on Diagnostic Testing for the Detection of Paratuberculosis in Cattle in the United States. Journal of the American Veterinary Medical Association. 229: 1912-1919.

11 Corner L.A.L., Gormley E. \& Pfeiffer D.U. 2012. Primary isolation of Mycobacterium bovis from bovine tissues: conditions for maximising the number of positive cultures. Veterinary Microbiology. 156 (1-2): 162-171.

12 De La Rua-Domenech R., Goodchild A.T., Vordermeier H.M., Hewinson R.G., Christiansen K.H. \& CliftonHadley R.S. 2006. Ante Mortem Diagnosis of Tuberculosis in Cattle: A Review of the Tuberculin Tests, -interferon Assay and other Ancillary Diagnostic Techniques. Research in Veterinary Science. 81(2): 190-210.

13 Fiss L., Santos B.L., Albuquerque P.P.F., Mota R.A., Marcolongo-Pereira C., Adrien M.L., Soares M.P. \& Schild A.L. 2015. Paratuberculose em Bovinos de Corte na Região Sul do Rio Grande do Sul. Pesquisa Veterinária Brasileira. 35(5): 437-442.

14 Gomes M.J.P., Driemeier D., Ribeiro V.R., Wunder Jr. E.A., Asanome W., Lanzon L.F. \& Wald V.B. 2002. Doença de Johne: Isolamento do Mycobacterium avium subsp. paratuberculosis (Map) em um rebanho leiteiro infectado na região Sul do Brasil. Acta Scientiae Veterinariae. 30(2): 113-118.

15 Larsen A.B., Merkal R.S. \& Cutlip R.C. 1975. Age of Cattle as Related to Resistence to Infection with Mycobacterium paratuberculosis. American Journal of Veterinary Research. 5: 255-257.

16 Menin A., Fleith R., Reck C., Marlow M., Fernandes P., Pilati C. \& Báfica A. 2013. Asymptomatic cattle naturally infected with Mycobacterium bovis present exacerbated tissue pathology and bacterial dissemination. Plos One. 8(1): e53884.

17 Monaghan M.L., Doherty M.L., Collins J.D., Kazda J.F. \& Quinn P.J. 1994. The Tuberculin Test. Veterinary Microbiology. 40(1-2): 111-124.

18 Mota R. A., Pinheiro-Junior J.W., Gomes M.J.P., Peixoto R.M., Maia F.C.I., Brito M.F. Chies J.A.B. Snel G.G.M., Bercht B.S. \& Juffo G.D. 2007. Paratuberculose em um Rebanho Leiteiro no Estado de Pernambuco, PE. Arquivos do Instituto Biológico. 74(2): 73-79.

19 Moura A., Hodon M.A., Soares Filho P.M., Issa M.A., Oliveira A.P.F. \& Fonseca Junior A.A. 2016. Comparison of nine DNA Extraction Methods for the Diagnosis of Bovine Tuberculosis by Real Time PCR. Ciência Rural. 46(7): 1223-1228.

20 Riet-Correa F. \& Driemeier D. 2007. Paratuberculose. In: Riet-Correa F., Schild A.L., Lemos R.A.A. \& Borges J.R.J. (Eds). Doenças de Ruminantes e Equinos. 3.ed. Santa Maria: Pallotti, pp.407-414.

21 Seva J., Sanes J.M., Ramis G., Mas A., Quereda J.J., Villareal-Ramos B., Villar D. \& Pallares F.J. 2014. Evaluation of the Single Cervical Skin Test and Interferon Gamma Responses to Detect Mycobacterium bovis Infected Cattle in a Herd Co-infected with Mycobacterium avium subsp. paratuberculosis. Veterinary Microbiology. 171: 139-146.

22 Taddei R., Barbieri I., Pacciarini M.L., Fallacara F., Belletti G.L. \& Arrigoni N. 2008. Mycobacterium porcinum Strains Isolated from Bovine Bulk Milk: Implications for Mycobacterium avium subsp. paratuberculosis Detection by PCR and culture. Veterinary Microbiology. 130: 338-347.

23 Timms V.J., Gehringer M.M., Mitchell H.M., Daskalopoulos G. \& Neilan B.A. 2011. Review. How Accurately can we Detect Mycobacterium avium subsp. paratuberculosis Infection? Journal of Microbiological Methods. 85(1): 1-8.

24 Verna A.E., Garcia-Pariente C., Muñoz M., Moreno O., García-Marin J.F., Romano M.I., Paolicchi F. \& Pérez V. 2007. Variation in the Immuno-pathological Responses of Lambs after Experimental Infection with Different Strains of Mycobacterium avium subsp. paratuberculosis. Zoonoses and Public Health. 54(6-7): 243-252.

25 Waters W.R., Buddle B.M., Vordermeier H.M., Gormley E., Palmer M.V., Thacker T.C., Bannantine J.P., Satbel J.R., Linscott R., Martel E., Milian F., Foshaug W. \& Lawrence J.C. 2011. Development and Evaluation of an Enzyme Linked Immunosorbent Assay for use in the Detection of Bovine Tuberculosis in Cattle. Clinical and Vaccine Immunology: CVI. 18(11): 1882-1888.

26 Welsh M.D., Cunningham R.T., Corbett D.M., Girvin R.M., McNair J., Skuce R.A., Bryson D.G. \& Pollock J.M. 2005. Influence of Pathological Progression on the Balance Between Cellular and Humoral Immune Responses in Bovine Tuberculosis. Immunology. 114(1): 101-111.

27 Yamasaki E.M., Tokarnia C.H., Galvão A., Gomes M.J.P., Chies J.A.B., Veit T.D., Aragão A.P. \& Brito M.F. 2010. Aspectos Clínico Patológicos e Controle da Paratuberculose em Rebanho Bovino Leiteiro. Pesquisa Veterinária Brasileira. 30(11): 921-932. 